\title{
Optic Neuritis as Isolated Manifestation of Leptomeningeal Carcinomatosis: A Case Report and Systematic Review of Ocular Manifestations of Neoplastic Meningitis
}

\author{
Silvia Lanfranconi, ${ }^{1}$ Paola Basilico, ${ }^{1}$ Ilaria Trezzi, ${ }^{1}$ Linda Borellini, ${ }^{1}$ Giulia Franco, ${ }^{1}$ \\ Vittorio Civelli, ${ }^{2}$ Francesco Pallotti, ${ }^{3}$ Nereo Bresolin, ${ }^{1}$ and Pierluigi Baron ${ }^{1}$ \\ ${ }^{1}$ Department of Neurological Sciences, Fondazione I.R.C.C.S. Ca' Granda Ospedale Maggiore Policlinico, Dino Ferrari Center, \\ 20122 Milan, Italy \\ ${ }^{2}$ Department of Neuroradiology and Interventional Neuroradiology, Fondazione I.R.C.C.S. Ca' Granda Ospedale Maggiore Policlinico, \\ 20122 Milan, Italy \\ ${ }^{3}$ Department of Pathological Anatomy, Fondazione Fondazione I.R.C.C.S. Ca' Granda Ospedale Maggiore Policlinico, \\ 20122 Milan, Italy
}

Correspondence should be addressed to Silvia Lanfranconi; silvialanfranconi@libero.it

Received 29 July 2013; Revised 30 August 2013; Accepted 31 August 2013

Academic Editor: Di Lazzaro Vincenzo

Copyright (C) 2013 Silvia Lanfranconi et al. This is an open access article distributed under the Creative Commons Attribution License, which permits unrestricted use, distribution, and reproduction in any medium, provided the original work is properly cited.

\begin{abstract}
Introduction. Leptomeningeal carcinomatosis occurs in about $5 \%$ of cancer patients. Ocular involvement is a common clinical manifestation and often the presenting clinical feature. Materials and Methods. We report the case of a 52-year old lady with optic neuritis as isolated manifestation of neoplastic meningitis and a review of ocular involvement in neoplastic meningitis. Ocular symptoms were the presenting clinical feature in 34 patients $(83 \%)$ out of 41 included in our review, the unique manifestation of meningeal carcinomatosis in 3 patients (7\%). Visual loss was the presenting clinical manifestation in 17 patients (50\%) and was the most common ocular symptom (70\%). Other ocular signs were diplopia, ptosis, papilledema, anisocoria, exophthalmos, orbital pain, scotomas, hemianopsia, and nystagmus. Associated clinical symptoms were headache, altered consciousness, meningism, limb weakness, ataxia, dizziness, seizures, and other cranial nerves involvement. All patients except five underwent CSF examination which was normal in 1 patient, pleocytosis was found in 11 patients, increased protein levels were observed in 16 patients, and decreased glucose levels were found in 8 patients. Cytology was positive in 29 patients (76\%). Conclusion. Meningeal carcinomatosis should be considered in patients with ocular symptoms even in the absence of other suggestive clinical symptoms.
\end{abstract}

\section{Introduction}

Leptomeningeal carcinomatosis results from dissemination of malignant cells to leptomeninges and can be observed in about $5 \%$ of patients with malignancies, but it is likely to become more frequent with the increase of life expectancy in cancer patients [1]. Neoplastic cells may spread to the subarachnoid space through (1) arterial circulation or, less frequently, through (2) retrograde flow in venous systems or (3) as a direct consequence of preexisting brain metastases or (4) through migration of neoplastic cells from the original tumor along perineural or perivascular spaces
$[2,3]$. Clinical manifestations can be highly variable and may affect both central (CNS) and peripheral nervous system (PNS). CNS involvement may lead to generalised symptoms such as seizures, confusion, encephalopathy, or intracranial hypertension as well as, less frequently, to focal neurological symptoms, mainly consisting in hemiparesis or aphasia. PNS involvement may present with lumbar and cervical radiculopathies or cranial neuropathies [2]. Ocular symptoms even in the absence of other clinical symptoms may represent the initial manifestation of meningeal carcinomatosis. Thus, meningeal carcinomatosis should be considered in the differential diagnosis in selected patients even if making 
the diagnosis is often challenging. Diagnostic tools consist mainly of contrast enhanced CT and MRI and lumbar puncture. Treatment options such as radiation and intrathecal chemotherapy are often palliative with an expected median patient survival of 2 to 6 months [2].

\section{Case Report}

A 52-year old lady was referred to our hospital for acute onset, ten days before hospitalization, of left orbital pain and visual loss associated with mild frontal throbbing headache. As symptoms progressed, she underwent ophthalmological evaluation as outpatient six days after symptoms onset, without evidence of significant visual loss and normal fundus oculi examination. Ocular computed tomography performed at that time was unremarkable. On subsequent ophthalmological evaluation five days later a significant visual loss in the left eye was evident with substantially normal fundus oculi examination. She underwent right mastectomy and hormonal therapy (tamoxifen) for infiltrative breast carcinoma in 2007 followed by chemotherapy with AC (cyclophosphamide and doxorubicin) for six months, followed by letrozole. She was on regular followup.

On admission in August 2011 neurological examination was normal except for mild anisocoria (left > right) with detectable Markus Gunn sign, left eye visual loss, and global reduction of deep tendon reflexes. She underwent contrastenhanced cerebral MRI showing nonspecific signal alteration involving frontal subcortical and periventricular white matter and focal contrast enhancement involving the left optic nerve sheath (see Figure 1). VEP revealed destructured response, reduced amplitude, and prolonged latency on the left (see Figure 2). Lumbar puncture revealed increased cell count (50 cells $/ \mathrm{mmc}$ ) with normal glucose and proteins. Oligoclonal bands were found on CSF but not in serum suggesting intrathecal immune response. Cytology at that time was negative for neoplastic cells and repeated ophthalmological evaluations revealed further progression to complete visual loss in the left eye. Serum antibodies (LAC, ACA, Anti-beta2glycoprotein ANA, anti-ds-DNA, ENA, c-ANCA, and pANCA) were negative. A diagnosis of possible inflammatory optic neuropathy was done. She was started on EV steroids followed by oral tapering without improvement.

In about two months she started to complain of left hearing loss. Brainstem auditory-evoked potentials were normal on the left (see Figure 2) and audiological evaluation evidenced mild neurosensorial failure more evident on the right. She underwent contrast-enhanced cerebral MRI in October 2011 showing increased enhancement of the left optic nerve sheath. Repeated ophthalmological evaluation revealed bilateral (left > right) papilledema and she was hospitalized. Neurological examination on admission showed total blindness in the left eye with absent pupillary response to direct light stimulation. VEP revealed absent response on the left and destructured response with normal amplitude and latency on the right (see Figure 2). Cerebral and spine MRI were unchanged except for nonspecific cerebellar enhancement. She underwent repeated lumbar puncture showing further increase of white cell count $(105$ cells/mmc) and reduced glucose. Citology was positive for atypical epithelial cells (see Figure 1). That was diagnostic for meningeal carcinomatosis. She was referred to the Oncology Department for further evaluation and treatment.

\section{Search Strategies and Methods}

References for this review were identified through a search of PubMed from 1966 to March 8, 2012 with the terms "meningeal carcinomatosis," "meningeal carcinomatosis and review," "meningeal carcinomatosis and optic neuritis," "meningeal carcinomatosis and ocular manifestations," and "neoplastic meningitis." Reference lists of relevant articles were also reviewed. Only articles with ocular manifestation as presenting or associated clinical features were included in our review.

\section{Results}

We found a total of 34 papers including 33 case reports (34 patients) and 1 case series (7 patients). Information about demographic details along with clinical, instrumental, and radiological findings were recorded and summarized in Table 1. In details we looked at latency between symptoms onset and diagnosis of meningeal carcinomatosis: the mean time was 4 months, while the mean time between primary tumor diagnosis and carcinomatosis diagnosis was 20 months. Six patients (14\%) had a postmortem diagnosis.

Ocular symptoms were the presenting clinical feature in 34 out of 41 patients $(83 \%)$ and were the only manifestation of meningeal carcinomatosis in 3 patients (7\%). Visual loss was the presenting clinical manifestation in 17 patients (50\%) and was the most common ocular symptom (70\%). Visual loss was bilateral in 8 patients and unilateral in 2 patients. Sequential optic nerve involvement was observed in 5 patients. Three patients had sudden onset of blindness. Visual loss was progressive in other six patients.

Additional ocular manifestations were diplopia (18 patients, presenting manifestation in 16 patients), ptosis (8 patients), papilledema (4), anysocoria (3), exophthalmos (2), orbital pain (2), scotomas (2), hemianopsia (1), and nystagmus (1).

Other common symptoms were headache (24 patients), altered consciousness (10 patients), meningism (8 patients), hemiparesis (7), ataxia (7), dizziness (6), and seizures (4). V (4), VII (6), VIII (4), IX and X (2), and XII (1) cranial nerves involvement has also been reported.

Twenty-three patients underwent enhanced MRI which was diagnostic in 8 patients (35\%). In 7 patients generalized meningeal enhancement was noted, and in 2 patients meningeal enhancement was associated with optic nerves enhancement, which was bilateral in 1 patient and unilateral in the other one. Three patients had cranial nerves enhancement, while isolated optic nerve enhancement was seen in only 1 patient (unilateral on the first MRI and bilateral at the second MRI). Other MRI findings included hydrocephalus (1), infarction of basal nuclei (1), and cerebellar enhancement (3). 


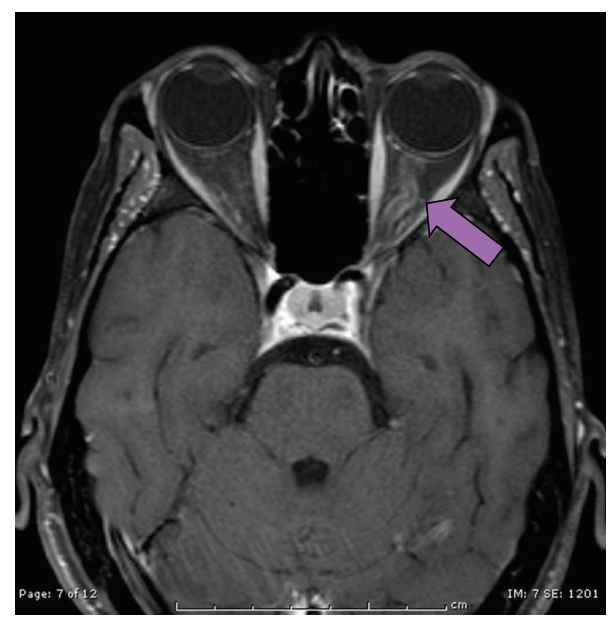

(a)

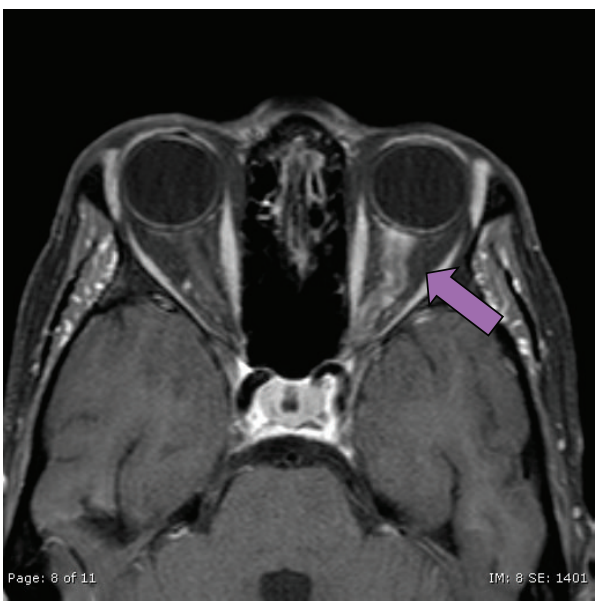

(c)

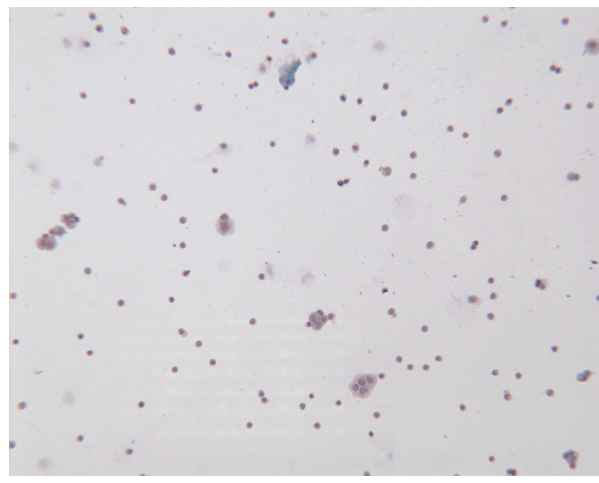

(e)

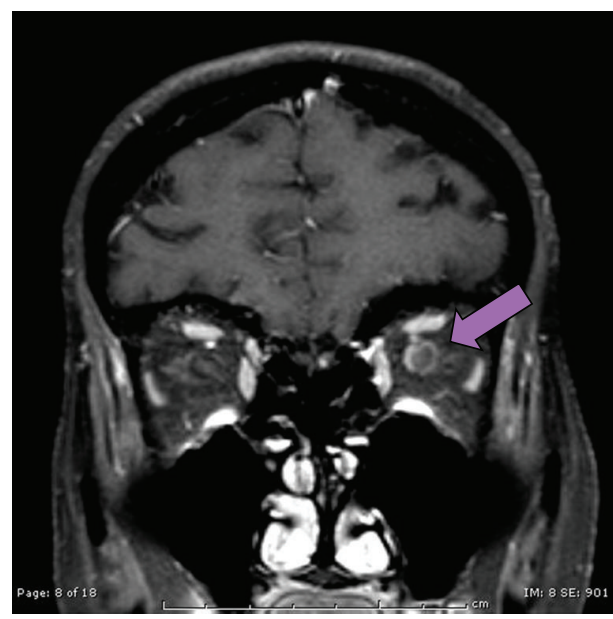

(b)

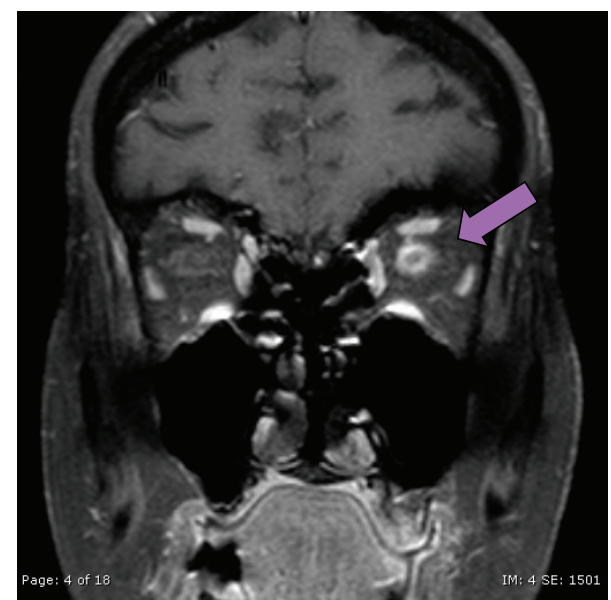

(d)

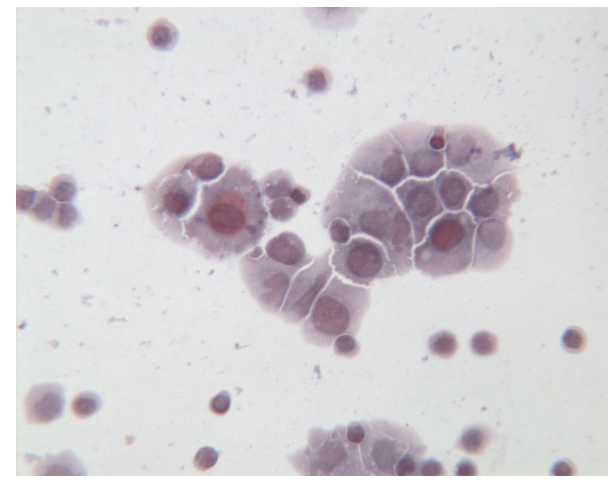

(f)

FIGURE 1: ((a)-(d)) Contrast-enhanced cerebral MRI showing diffuse enhancement of the left optic nerve at onset ((a), axial T1; (b), coronal T1) and at 2 months followup ((c), axial T1_SE_FSAT; (d) coronal T1_SE_FSAT). ((e)-(f)) Cerebrospinal fluid cytology showing atypical epithelial cells aggregates.

8 patients had normal MRI findings.

All patients except five underwent CSF examination which was normal in 1 patient, and increased cells count was found in 11 patients (range: $9-598 / \mathrm{mm}^{3}$ ), increased protein levels were observed in 16 patients (range: $62-334 \mathrm{mg} / \mathrm{dL}$ ), and decreased glucose levels were found in 8 patients (range: $5-43 \mathrm{mg} / \mathrm{dL}$ ). Citology was positive in 29 patients $(76 \%)$.
The histology of the original tumor was highly variable with solid tumors being more frequently associated with meningeal carcinomatosis. The most common was gastric cancer followed by lymphoma, breast, and lung cancer.

16 patients underwent chemotherapy (see Table 1), and the mean life expectancy was 123 days (range 5-730 days). Autopsy was obtained in 13 patients. See Table 1. 


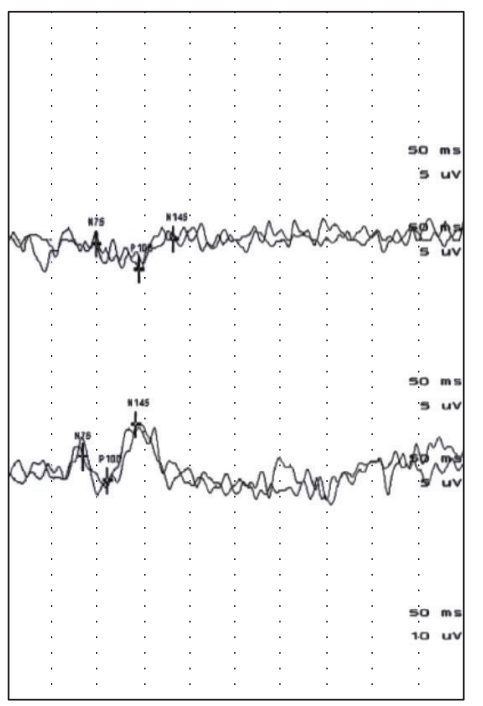

(a)

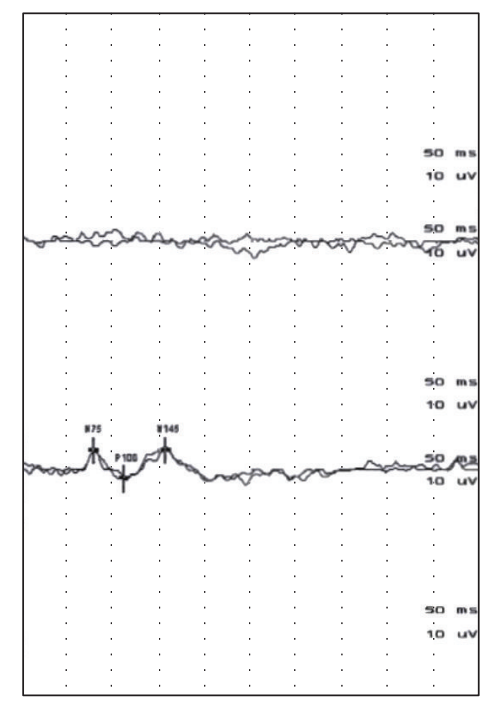

(c)

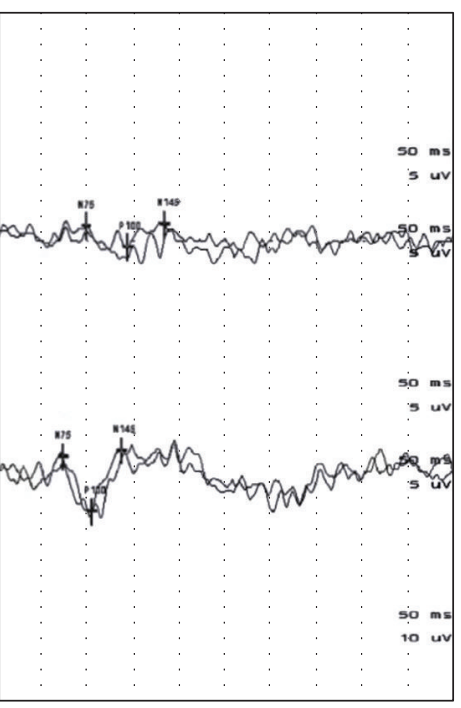

(b)

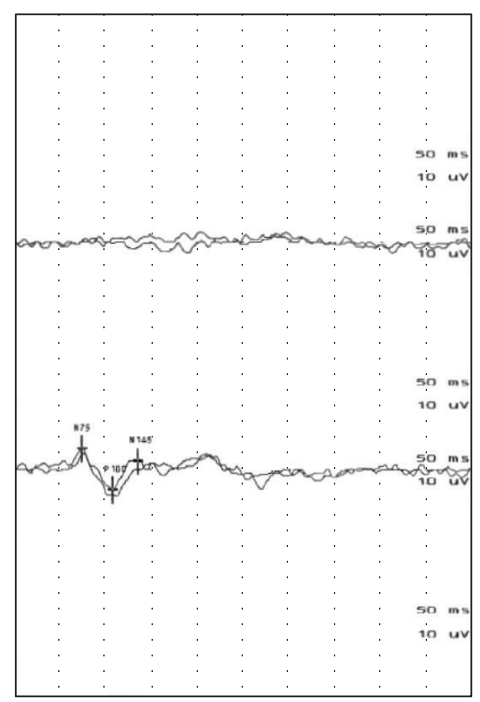

(d)

FIGURE 2: Visual evoked potentials (VEPs) at onset ((a), (b)) and at two months follow-up ((c), (d)). (a)-(b): Response at 15 (a) and 30 (b) sec showing destructured response, reduced amplitude and prolonged latency on the left and normal response on the right side. (c)-(d): Response at 15 (c) and 30 (d) sec revealing absent response on the left and destructured response with normal amplitude and latency on the right side.

\section{Discussion}

Meninigeal carcinomatosis occurs in $1-5 \%$ of solid tumors being adenocarcinoma the most common histology. The highest leptomeningeal diffusion rate has been reported for small cell lung cancer (11\%) and melanoma (20\%). Meningeal involvement occurs in about $5 \%$ of breast cancer patients [1]. Neoplastic meningitis is more likely to occur in patients with disseminated cancer, but in, respectively, $20 \%$ and $10 \%$ of cases it may manifest after a disease-free period or as a first manifestation of systemic tumors [2]. Current diagnostic methods are limited and may fail to identify MC early enough to prevent the escalation of neurological damage. Thus meningeal carcinomatosis should be included in the differential diagnosis in the presence of multifocal disease but also considered in the evaluation of isolated syndromes such as intracranial hypertension, cauda equine syndrome, or cranial neuropathy. The most common manifestation of cranial nerves involvement is diplopia due to VI cranial nerve palsy, followed by III and IV involvement. V, VIII, and optic nerve may also be affected [38].

Diagnostic workup includes CSF examination and neuroradiological studies. The presence of malignant cells in the CSF is diagnostic, other supportive features are increased opening pressure, pleocytosis, elevated proteins, and decreased glucose levels. CSF cytology may be negative in $65 \%$ of patients on initial examination, but only in $20 \%$ of patients on second lumbar puncture [2]. CSF increased levels of vascular endothelial growth factors have been proposed as a promising biomarker with a reported sensitivity of $51-100 \%$ 


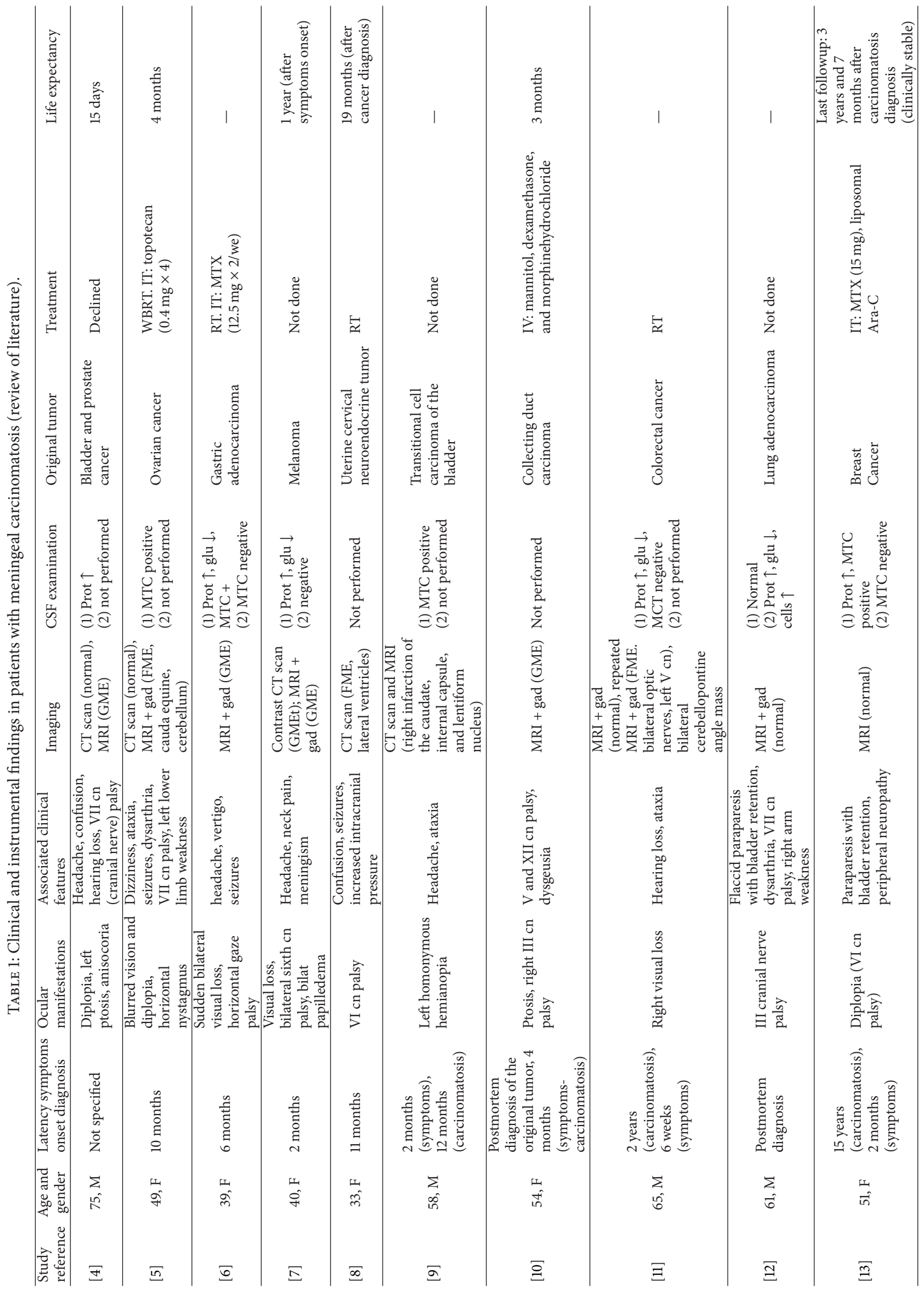




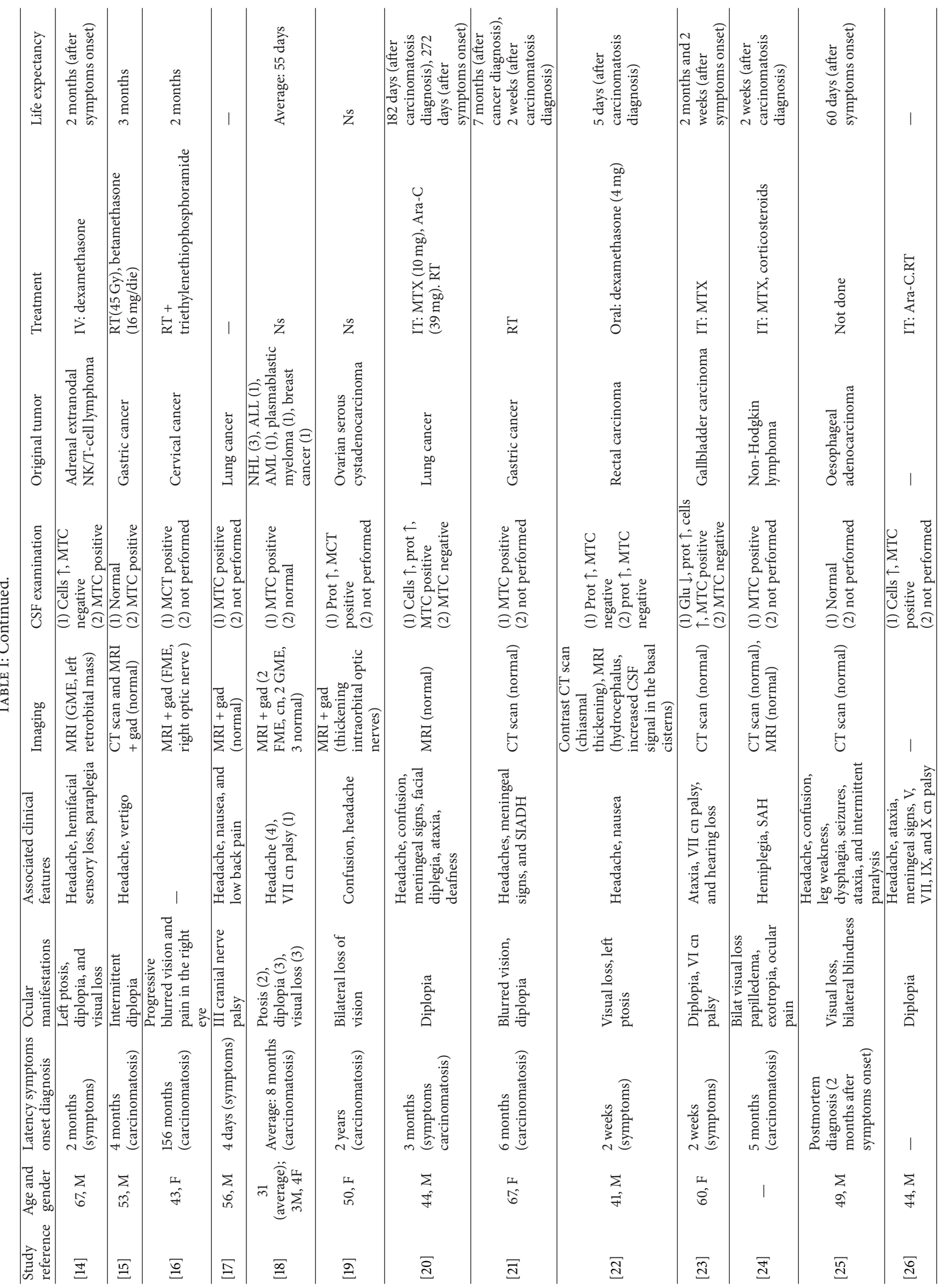




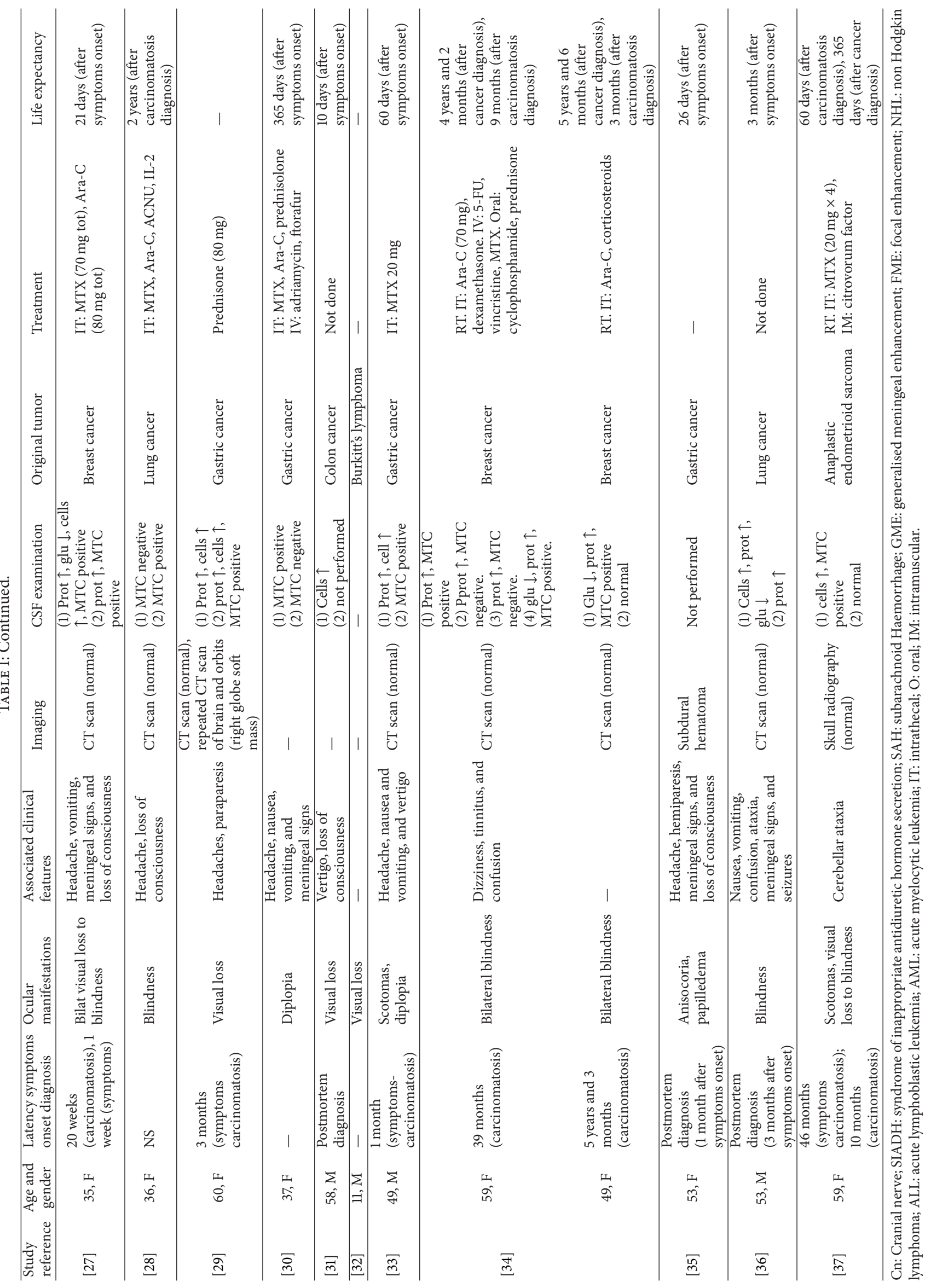


and a specificity of $73-100 \%$ for leptomeningeal metastases [39].

Gadolinium-enhanced MRI is also useful for the diagnosis of meningeal carcinomatosis because enhancement on MRI will reveal any irritation of leptomeninges resulting in cranial nerves or intradural extramedullary enhancement on spinal MRI.

Ocular involvement represents a frequent manifestation of meningeal carcinomatosis. The reported frequency of ocular signs may be as high as $90 \%$ [40]. The present report is an updated and systematic review of ocular manifestations of neoplastic meningitis. Our data show that ocular symptoms often represent the first clinical manifestation of meningeal carcinomatosis $(83 \%)$ and the only clinical manifestation in a small proportion of subjects (7\%). For this reason it should be considered in the differential diagnosis even in the absence of associated clinical symptoms more suggestive of meningeal carcinomatosis such as headache (58\%), altered consciousness (24\%), meningism (19\%), focal signs (34\%), dizziness $(15 \%)$, seizures (10\%), and cranial nerves other than ocular involvement (32\%). The most common ocular manifestation was visual loss (70\%) followed by diplopia (41\%), ptosis (19\%), papilledema (10\%), anysocoria (7\%), exophthalmos (5\%), orbital pain (5\%), scotomas (5\%), hemianopsia (2\%) and nystagmus (2\%). Gadolinium-enhanced MRI was diagnostic only in about one third of patients. Meningeal enhancement was detected in about one third of patients; in two of them (25\%) it was associated with focal optic nerve enhancement. In one patient (12\%) contrastenhanced MRI revealed isolated optic nerve enhancement. CSF examination was abnormal in all (36) but one patient. The most common finding was increased proteins level (44\%), increased cells count (30\%), and decreased glucose (22\%). Citology was positive in a high proportion of patients (76\%) with solid tumors being the more frequent. The first CSF examination may be inconclusive, thus if clinical and radiological suspicion persist and cell count is increased, a repeated lumbar puncture is recommended.

\section{Conclusion}

Ocular involvement is a frequent and early clinical manifestation of meningeal carcinomatosis. Moreover clinicians should be aware that ocular involvement may mimic different diseases as shown in our case report, where neoplastic optic nerve involvement was indistinguishable from optic neuritis. Thus meningeal carcinomatosis should be included in the differential diagnosis of diplopia and visual loss in selected patients because diagnosis is often challenging.

\section{Conflict of Interests}

The authors have no conflict of interests to declare.

\section{References}

[1] M. C. Chamberlain, "Carcinomatous meningitis," Archives of Neurology, vol. 54, no. 1, pp. 16-17, 1997.
[2] M. C. Chamberlain, "Neoplastic meningitis," Oncologist, vol. 13, no. 9, pp. 967-977, 2008.

[3] S. A. Grossman and M. J. Krabak, "Leptomeningeal carcinomatosis," Cancer Treatment Reviews, vol. 25, no. 2, pp. 103-119, 1999.

[4] J. Walz, "Ocular manifestations of meningeal carcinomatosis: a case report and literature review," Optometry, vol. 82, no. 7, pp. 408-412, 2011.

[5] E. Miller, I. Dy, and T. Herzog, "Leptomeningeal carcinomatosis from ovarian cancer," Medical Oncology, vol. 29, no. 3, pp. 20102015, 2012.

[6] G. Bulut, A. Erden, B. Karaca, and E. Göker, "Leptomeningeal carcinomatosis of gastric adenocarcinoma," Turkish Journal of Gastroenterology, vol. 22, no. 2, pp. 195-198, 2011.

[7] M. Arias, M. Alberte-Woodward, S. Arias, D. Dapena, Á. Prieto, and J. M. Suárez-Peñaranda, "Primary malignant meningeal melanomatosis: a clinical, radiological and pathologic case study," Acta Neurologica Belgica, vol. 111, no. 3, pp. 228-231, 2011.

[8] S. Komiyama, E. Nishio, Y. Torii et al., "A case of primary uterine cervical neuroendocrine tumor with meningeal carcinomatosis confirmed by diagnostic imaging and autopsy," International Journal of Clinical Oncology, vol. 16, no. 5, pp. 581-586, 2011.

[9] C. Butchart, A. Dahle-Smith, D. Bissett, J. M. Mackenzie, and D. J. Williams, "Isolated meningeal recurrence of transitional cell carcinoma of the bladder," Case Reports in Oncology, vol. 3, no. 2, pp. 171-175, 2010.

[10] S. Ohnishi, M. Dazai, Y. Iwasaki, K. Tsuzaka, T. Takahashi, and T. Miyagishima, "Undiagnosed collecting duct carcinoma presenting as meningeal carcinomatosis and multiple bone metastases," Internal Medicine, vol. 49, no. 15, pp. 1541-1544, 2010.

[11] B. B. Bruce, M. Tehrani, N. J. Newman, and V. Biousse, "Deafness and blindness as a presentation of colorectal meningeal carcinomatosis," Clinical Advances in Hematology and Oncology, vol. 8, no. 8, pp. 564-566, 2010.

[12] K. L. Breivik, R. Laurini, R. Steen, and K. B. Alstadhaug, "A 61-year-old man with sciatica," Tidsskrift for den Norske Loegeforening, vol. 129, no. 10, pp. 1000-1002, 2009.

[13] P. Gaviani, A. Silvani, E. Corsini, A. Erbetta, and A. Salmaggi, "Neoplastic meningitis from breast carcinoma with complete response to liposomal cytarabine: case report," Neurological Sciences, vol. 30, no. 3, pp. 251-254, 2009.

[14] K. K. Dunning, K. Wudhikarn, A.-O. Safo, C. J. Holman, R. W. McKenna, and S. E. Pambuccian, "Adrenal extranodal NK/T-cell lymphoma diagnosed by fine-needle aspiration and cerebrospinal fluid cytology and immunophenotyping: a case report," Diagnostic Cytopathology, vol. 37, no. 9, pp. 686-695, 2009.

[15] T. Yamada, K. Furukawa, K. Yokoi, Y. Ohaki, S. Okada, and T. Tajiri, "A case of meningeal carcinomatosis with gastric cancer which manifested meningeal signs as the initial symptom; the palliative benefit of radiotherapy," Journal of Nippon Medical School, vol. 75, no. 4, pp. 216-220, 2008.

[16] C. C. Portera, R. F. Gottesman, M. Srodon, F. Asrari, M. Dillon, and D. K. Armstrong, "Optic neuropathy from metastatic squamous cell carcinoma of the cervix: an unusual CNS presentation," Gynecologic Oncology, vol. 102, no. 1, pp. 121-123, 2006.

[17] R. Nardone, M. Herz, E. Egarter-Vigl, and F. Tezzon, "Isolated oculomotor nerve palsy as the presenting clinical manifestation of a meningeal carcinomatosis: a case report," Neurological Sciences, vol. 27, no. 4, pp. 288-290, 2006. 
[18] M. Gokce, "Analysis of isolated cranial nerve manifestations in patients with cancer," Journal of Clinical Neuroscience, vol. 12, no. 8, pp. 882-885, 2005.

[19] J. Levy, M. Marcus, I. Shelef, and T. Lifshitz, "Acute bilateral blindness in meningeal carcinomatosis," Eye, vol. 18, no. 2, pp. 206-208, 2004.

[20] Y. Sato, Y. Ohta, M. Kaji, K. Oizumi, and M. Kaji, "Carbohydrate antigen 19-9 in cerebrospinal fluid and within malignant cells in a case of leptomeningeal carcinomatosis," Journal of Neurology Neurosurgery and Psychiatry, vol. 65, no. 3, pp. 402-403, 1998.

[21] M. Mouallem, N. Ela, and G. Segal-Lieberman, "Meningeal carcinomatosis and syndrome of inappropriate antidiuretic hormone in a patient with metastatic carcinoma of the stomach," Southern Medical Journal, vol. 91, no. 11, pp. 1076-1078, 1998.

[22] R. McFadzean, D. Brosnahan, D. Doyle, J. Going, D. Hadley, and W. Lee, "A diagnostic quartet in leptomeningeal infiltration of the optic nerve sheath," Journal of Neuro-Ophthalmology, vol. 14, no. 3, pp. 175-182, 1994.

[23] R. J. J. Tans, J. Koudstaal, and P. J. Koehler, "Meningeal carcinomatosis as presenting symptom of a gallbladder carcinoma," Clinical Neurology and Neurosurgery, vol. 95, no. 3, pp. 253-256, 1993.

[24] A. Neetens, A. Clemens, P. van den Ende, R. de Bock, and I. Neetens, "Optic neuritis in non Hodgkin lymphomas," Klinische Monatsblatter fur Augenheilkunde, vol. 200, no. 5, pp. 525-528, 1992.

[25] J. P. Teare, M. Whitehead, M. O. Rake, and R. J. Coker, "Rapid onset of blindness due to meningeal carcinomatosis from an oesophageal adenocarcinoma," Postgraduate Medical Journal, vol. 67, no. 792, pp. 909-911, 1991.

[26] Y. Sato, Y. Ohta, T. Ohtsuka, H. Shoji, and K. Oizumi, "A case of leptomeningeal carcinomatosis: demonstration of CA19-9 and CEA positive malignant cells in the CSF and particular elevation of CA19-9 level in the CSF," Rinshō Shinkeigaku, vol. 31, no. 2, pp. 175-178, 1991.

[27] W. Boogerd, D. Moffie, and L. A. Smets, "Early blindness and coma during intrathecal chemotherapy for meningeal carcinomatosis," Cancer, vol. 65, no. 3, pp. 452-457, 1990.

[28] A. Watanabe, M. Mizobe, Y. Ogawa et al., "A case of longterm survival of a patient with complicated diffuse metastatic leptomeningeal carcinomatosis secondary to lung adenocarcinoma," Nihon Kyobu Shikkan Gakkai Zasshi, vol. 28, no. 8, pp. 1130-1135, 1990.

[29] J. A. McCrary III, J. R. Patrinely, and R. L. Font, "Progressive blindness caused by metastatic occult signet-ring cell gastric carcinoma," Archives of Ophthalmology, vol. 104, no. 3, pp. 410413, 1986.

[30] N. Usui, M. Ogawa, J. Inagaki et al., "Case report of meningeal carcinomatosis of gastric cancer successfully treated with intrathecal and systemic chemotherapy," Gan to Kagaku Ryoho, vol. 12, no. 1, pp. 155-159, 1985.

[31] H. Ohkawa, K. Nakazawa, K. Nakada, and J. Miyauchi, "Carcinoma of the colon associated with diffuse metastatic leptomeningeal carcinomatosis (DMLC) presenting as disturbance of consciousness-a case report," Gan No Rinsho, vol. 30, no. 4, pp. 409-412, 1984.

[32] L. A. Donoso, L. E. Magargal, and R. A. Eiferman, "Meningeal carcinomatosis secondary to malignant lymphoma (Burkitt's pattern)," Journal of Pediatric Ophthalmology and Strabismus, vol. 18, no. 1, pp. 48-50, 1981.
[33] G. Agnelli and P. Gresele, "Mucus-secreting 'signet-ring' cells in CSF revealing the site of primary cancer," Postgraduate Medical Journal, vol. 56, no. 662, pp. 868-870, 1980.

[34] R. Cantillo, J. Jain, A. Singhakowinta, and V. K. Vaitkevicius, "Blindness as initial manifestation of meningeal carcinomatosis in breast cancer," Cancer, vol. 44, no. 2, pp. 755-757, 1979.

[35] S. Sakaki, Y. Mori, K. Matsuoka, T. Ohnishi, and S. Bitoh, "Metastatic dural carcinomatosis secondary to gastric cancer," Neurologia Medico-Chirurgica, vol. 19, no. 1, pp. 39-44, 1979.

[36] R. E. Appen, G. de Venecia, J. H. Selliken, and L. T. Giles, "Meningeal carcinomatosis with blindness," American Journal of Ophthalmology, vol. 86, no. 5, pp. 661-665, 1978.

[37] P. H. Altrocchi and P. B. Eckman, "Meningeal carcinomatosis and blindness," Journal of Neurology Neurosurgery and Psychiatry, vol. 36, no. 2, pp. 206-210, 1973.

[38] M. Balm and J. Hammack, "Leptomeningeal carcinomatosis: presenting features and prognostic factors," Archives of Neurology, vol. 53, no. 7, pp. 626-632, 1996.

[39] F. Deisenhammer, R. Egg, G. Giovannoni et al., "EFNS guidelines on disease-specific CSF investigations," European Journal of Neurology, vol. 16, no. 6, pp. 760-770, 2009.

[40] W. R. Wasserstrom, J. P. Glass, and J. B. Posner, "Diagnosis and treatment of leptomeningeal metastases from solid tumors: experience with 90 patients," Cancer, vol. 49, no. 4, pp. 759-772, 1982. 


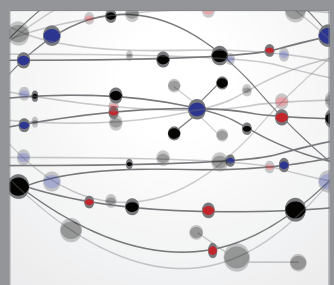

The Scientific World Journal
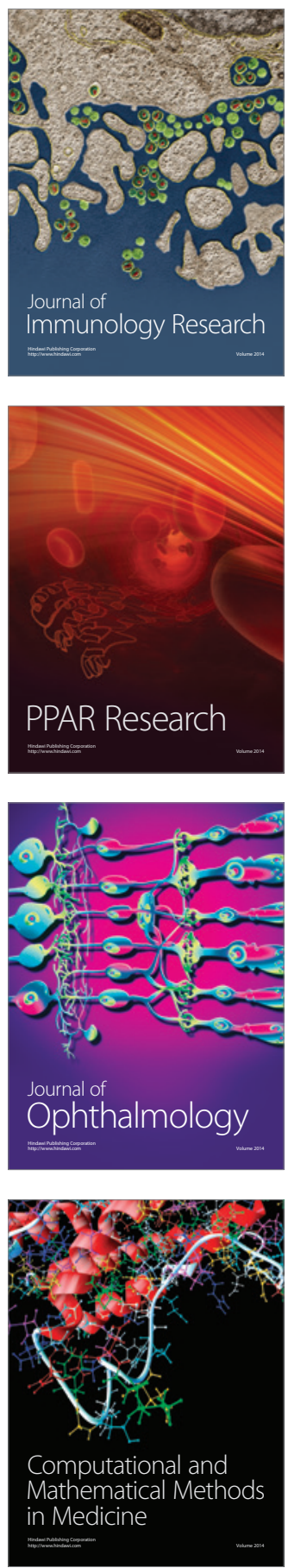

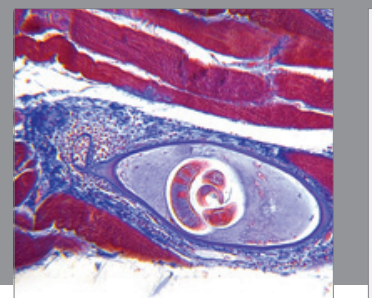

Gastroenterology

Research and Practice
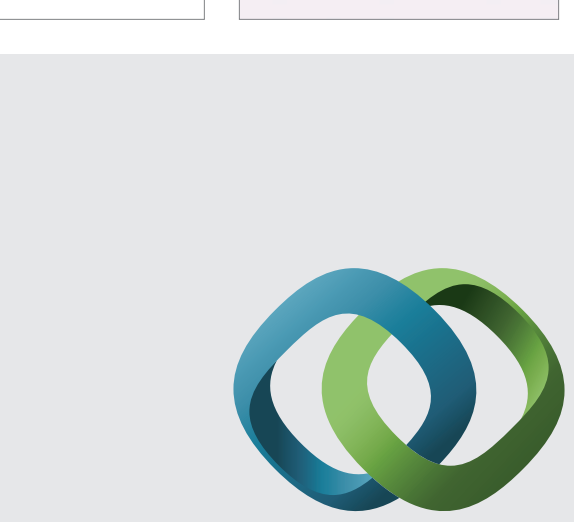

\section{Hindawi}

Submit your manuscripts at

http://www.hindawi.com
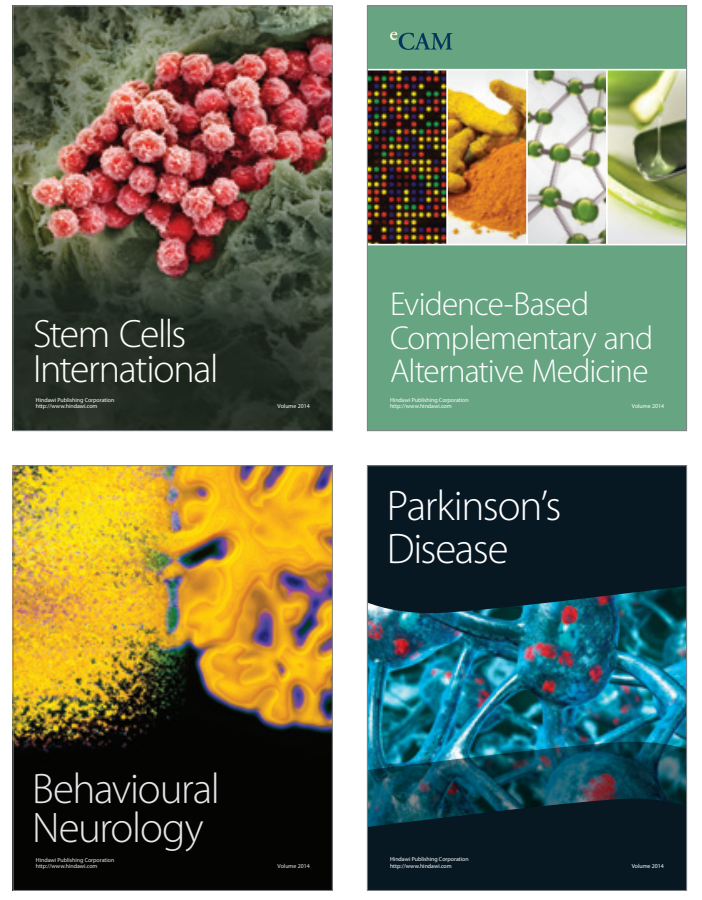
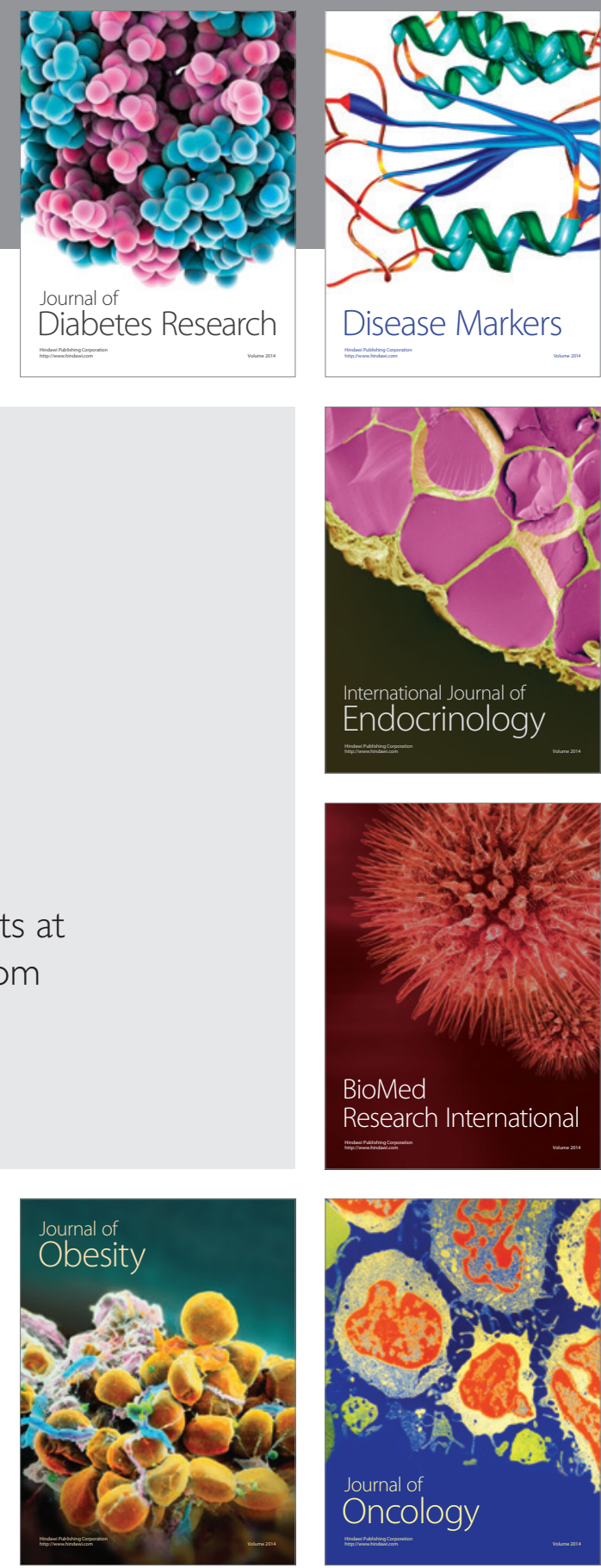

Disease Markers
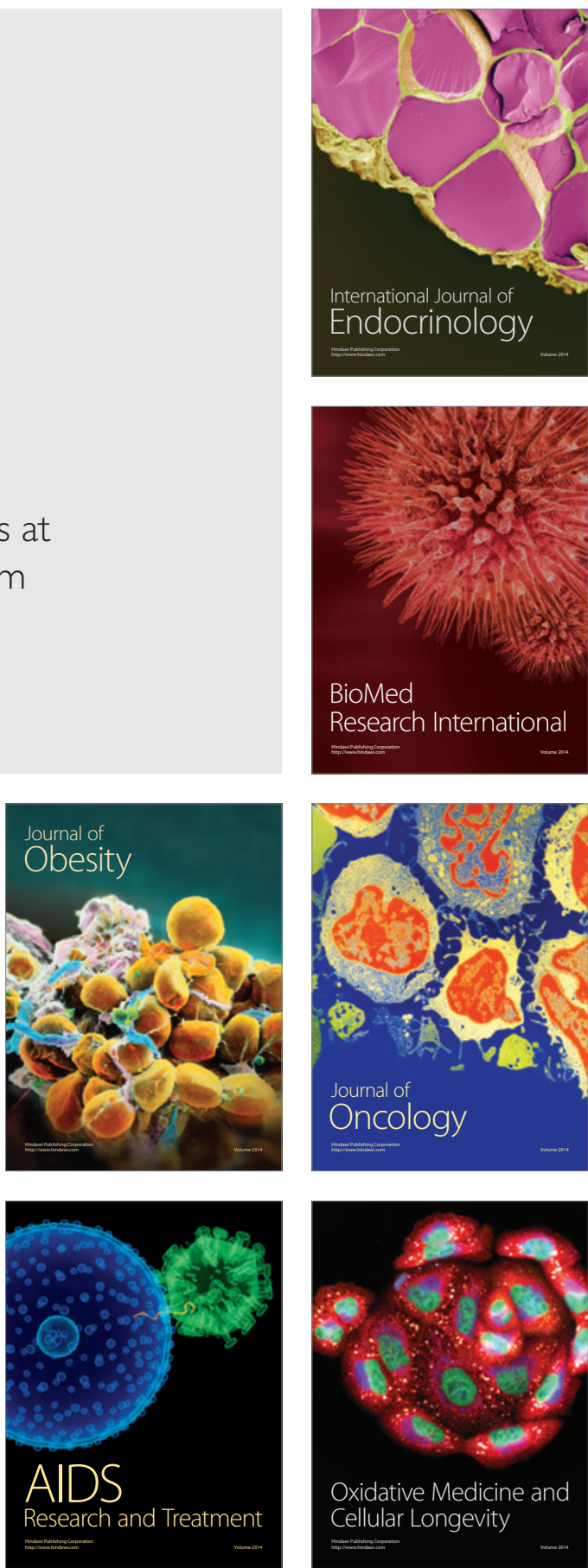